\title{
Les volailles pour un contrôle biologique des adventices dans les vergers
}

\author{
Anaïs LAVIGNE ${ }^{1 *}$, Eddy Dumbardon-MARTIAL ${ }^{1}$, Christian LAVIGNE $^{2,3}$
}

${ }^{1}$ FREDON, Route du Lycée Agricole, Croix Rivail, 97224 Ducos, Martinique, a.lavigne@fredon972.fr

2 CIRAD-Persyst, TA B-103/ PS4, Blvd. La Lironde, 34398 Montpellier Cedex 5, France

3 PRAM, Petit Morne, 97285 Lamentin, Martinique

${ }^{*}$ Correspondance et tirés-à-part

Reçu le 19 octobre 2011

Accepté le 18 novembre 2011

Fruits, 2012, vol. 67, p. 341-351 (C) 2012 Cirad/EDP Sciences All rights reserved DOI: 10.1051/fruits/2012029 Www.fruits-journal.org

RESUMEN EsPañol, p. 351

\section{Poultry for biological control of weeds in orchards.}

Abstract - Introduction. Because of the humid tropical conditions, weed control is an important and permanent job in the outlay of a fruit producer in the West Indies. The practice of chemical weed control is to date the cheapest, but the recurring use of herbicides in the orchards of Martinique leads to soil erosion and water pollution. To mitigate the use of herbicides, we tested the association of poultry with a productive orchard in order to control the weeds. Materials and methods. The system was installed on a plot of $700 \mathrm{~m}^{2}$ divided into seven plots of $100 \mathrm{~m}^{2}$. Two plots were the control treatments and were mowed with a brush cutter. Each of the other five plots was subjected to five geese grazing for a week, then to 20 chickens grazing during the following week, then the plot was left without poultry for three more weeks. Three cycles of such rotations were observed ( 4 months). Biomass production by botanical groups (Poaceae, Cyperaceae and others) in the experimental plots was measured each week for four months. A monthly index of recovery by botanical group was noted. Results. The total biomass of the herbaceous cover decreased on grazed plots, which allowed the biological control of weeds for four months. Species diversity decreased because of the selection made by poultry for food and cover was homogenized by the percentage increase in biomass of Cyperaceae in the total biomass. This group, the least palatable for poultry, thus flourished on all plots grazed. Discussion. Poultry grazing, particularly geese, proved to be a promising method for controlling weeds in orchards. However, in the case of a high proportion of unpalatable species in the herbaceous cover, other weed control methods should be combined. The installation of a grass cover composed of a mixture of cover crops adapted to the combination of orchard and poultry as well as better management of the animal rotation should be considered to optimize this technique.

Martinique / Psidium guajava / orchards / perennials / cultivation / integrated control / weed control / geese / chickens

\section{Les volailles pour un contrôle biologique des adventices dans les vergers.}

Résumé - Introduction. Du fait des conditions tropicales humides, le contrôle des adventices est un poste important et permanent dans les dépenses d'un producteur de fruits aux Antilles. La pratique du désherbage chimique est à ce jour la plus économique, mais l'utilisation récurrente d'herbicides dans les vergers de la Martinique conduit à une érosion des sols et à une pollution des eaux. Pour pallier l'usage d'herbicides, nous avons testé l'association de volailles à un verger productif afin d'en contrôler l'enherbement. Matériel et méthodes. Le dispositif a été installé sur une parcelle de $700 \mathrm{~m}^{2}$ subdivisée en sept placettes de $100 \mathrm{~m}^{2}$. Deux placettes ont constitué les traitements témoins ; elles ont été fauchées à la débroussailleuse. Chacune des cinq autres placettes a été soumise au pâturage de 5 oies pendant une semaine, puis au pâturage de 20 poulets pendant la semaine suivante, puis laissée sans volailles pendant trois autres semaines. Trois cycles de telles rotations ont été observés. La production de biomasse par groupe botanique (poacées, cypéracées et autres) dans les placettes expérimentales a été mesurée chaque semaine pendant les trois cycles (4 mois). Une fois par mois l'indice de recouvrement par groupe botanique a été relevé. Résultats. La biomasse totale de la strate herbacée a diminué sur les placettes pâturées, ce qui a permis le contrôle biologique des adventices pendant quatre mois. La diversité floristique a diminué à cause de la sélection opérée par les volailles pour leur alimentation et le couvert s'est homogénéisé par l'augmentation du pourcentage de la biomasse des cypéracées dans la biomasse totale. Cette famille peu appétible pour les volailles s'est ainsi étendue sur toutes les placettes pâturées. Discussion. Le pâturage des volailles, des oies en particulier, s'est révélé être une méthode efficace pour la maîtrise de l'enherbement en verger. Cependant, dans le cas d'une forte proportion d'espèces non appétibles dans la strate herbacée, d'autres méthodes de désherbage devront être combinées. L'installation d'une couverture herbacée composée d'un mélange de plantes de couverture adaptées à l'association verger et volailles ainsi qu'une meilleure gestion de la rotation des animaux devront être envisagées pour optimiser cette technique.

Martinique / Psidium guajava / verger / plante pérenne / pratique culturale / lutte intégrée / désherbage / oie / poulet 


\section{Introduction}

Aux Antilles, le contrôle de l'enherbement constitue l'une des contraintes majeures de la conduite des vergers. Les conditions climatiques (température et pluie) restent favorables au développement des adventices pendant une très grande partie de l'année. Le choix du désherbage chimique par les agriculteurs conventionnels est motivé par la difficulté de mécanisation de leurs exploitations. Le faible coût du désherbage chimique tant en main-d'œuvre qu'en intrants contribue également à l'utilisation de cette méthode qui reste encore la plus courante.

Par ailleurs, des résidus d'herbicides sont régulièrement trouvés dans les eaux de rivière, en particulier des résidus de glyphosate et de son métabolite l'acide aminomethyl-phosphonique (AMPA) [1]. L'utilisation excessive d'herbicides en agriculture conduit en outre à l'apparition de phénomènes d'érosion et de lessivage des sols [2, 3]. Les vergers pentus y sont donc particulièrement sensibles.

Les agriculteurs adeptes d'une agriculture biologique et les agriculteurs désireux de diminuer l'emploi des herbicides ne possèdent pas d'autres méthodes de désherbage réellement satisfaisantes : le gyrobroyage est rarement mis en œuvre du fait de la forte déclivité des terrains et l'utilisation de la débroussailleuse, même si elle est privilégiée dans certaines situations, reste une opération fastidieuse et pénible qui représente un coût important en main-d'œuvre.

Une solution pour remplacer le désherbage chimique consiste à associer production fruitière et élevage, le pâturage des animaux permettant de désherber le verger. Il existe de nombreux ouvrages concernant les recherches sur les systèmes sylvopastoraux utilisant des ruminants [4]. De tels systèmes ont obtenu des résultats encourageants aussi bien en zone tempérée [5, 6] qu'en zone tropicale où l'association entre sylviculture et pâturage de bovins est largement pratiquée [7].

Si associer pâturage et production forestière est relativement fréquent, associer pâturage de ruminants et production frui- tière est beaucoup plus anecdotique voire proscrit, les animaux ne devant pas porter préjudice aux cultures [8]. En effet les ruminants causent de nombreux dégâts sur les branches basses des arbres et sont susceptibles de consommer les feuilles et les fruits. Associer volailles et production fruitière présente moins de risques, mais les études portant sur ce type de systèmes sont moins nombreuses. Pourtant, intégrer des volailles dans un système agro-forestier présente certains intérêts que ce soit en termes de bienêtre animal ou d'économie de foncier [9] que de contrôle des ravageurs [10, 11] et des mauvaises herbes [11, 12].

Des poulets et des oies élevés en plein air dans un verger de pommiers associé à des plants de pommes de terre ont permis à la fois de diminuer l'abondance de certains insectes ravageurs (grâce aux poulets pour une surface de 18 à $34 \mathrm{~m}^{2}$ par poulet) et de contrôler l'enherbement (grâce aux oies pour une surface de $48 \mathrm{~m}^{2}$ par oie) [10]. Bien que moins efficaces en termes de désherbage pour des densités inférieures à 910 poulets par ha [13], les poulets permettent de lutter contre certaines adventices telles que Cyperus rotundus dans de petits enclos [11] et de diminuer les besoins en désherbage [12]. Pour ce qui est du désherbage, les oies sont plus efficaces car parfaitement herbivores, alors que les poulets sont granivores; elles ont longtemps été utilisées pour contrôler l'enherbement dans des champs de coton [11, 14, 15]. Elles possèdent un fort potentiel pour une stratégie de gestion intégrée des adventices dans les vergers en zone tempérée [10, 14, 16]. À notre connaissance, aucune étude récente associant une production fruitière et un élevage de volailles n'a été conduite en zone tropicale. Il existe donc peu de références quant à la gestion des volailles élevées sur un parcours planté pour maîtriser l'enherbement.

Par ailleurs, si le producteur souhaitait dans le passé éliminer totalement la couverture herbacée pour éviter toute concurrence entre espèces végétales, il cherche maintenant à conserver un couvert herbacé afin de limiter les phénomènes d'érosion et de lessivage. Cette couverture du sol doit pouvoir être facilement contrôlée pour permettre les 
activités culturales en verger comme la taille et la récolte. Pour tenter de réduire l'utilisation des herbicides en cultures pérennes, nous avons testé, chez un arboriculteur, la possibilité d'associer une production de goyaves destinées à la transformation à un élevage de volailles; des oies ont été utilisées du fait de leur régime alimentaire herbivore et donc de leur capacité de désherbage [16], et des poulets ont également été considérés pour gérer les espèces refusées par les oies [11, 17] et consommer les fruits tombés au sol, tout en améliorant la rentabilité du système par la production d'œufs et de viande [12].

L'objectif principal de notre essai a été de vérifier que l'enherbement d'une parcelle fruitière peut être contrôlé par un élevage de volailles, entrainant une diminution du nombre de fauches mécaniques à la débroussailleuse.

\section{Matériel et méthodes}

\subsection{Dispositif expérimental}

L'essai a été mené d'août à décembre 2010 chez un agriculteur de la commune du SaintEsprit à la Martinique (14 33' 37" lat. N, $60^{\circ} 55^{\prime}$ 05" long. O, alt. $\left.35 \mathrm{~m}\right)$. Le sol est composé d'alluvions lourdes montmorillonitiques [18]. La température moyenne annuelle en Martinique en 2010 a été de $27,6{ }^{\circ} \mathrm{C}^{1}$, avec une moyenne des valeurs maximales de $33,9^{\circ} \mathrm{C}$ en février et minimales de $20,0{ }^{\circ} \mathrm{C}$ en octobre ${ }^{2}$. Durant cette même année, la pluviosité a été de $2337 \mathrm{~mm}$ au Saint-Esprit ${ }^{2}$.

Le dispositif a été installé sur un verger de goyaviers de la variété "Beaumont "dont les arbres sont productifs, régulièrement taillés,

\footnotetext{
${ }^{1}$ Bernard E., Bilan climatique annuel 2010 en Martinique, Météo-France, http://www. meteo.fr/temps/domtom/antilles/packpublic/alaune/bca_2010_martinique.pdf.

${ }^{2}$ Les données météorologiques, Conseil général de la Martinique, Services techniques et économiques, 2010, http://www. cgste.mq/DonneesMeteo/
}

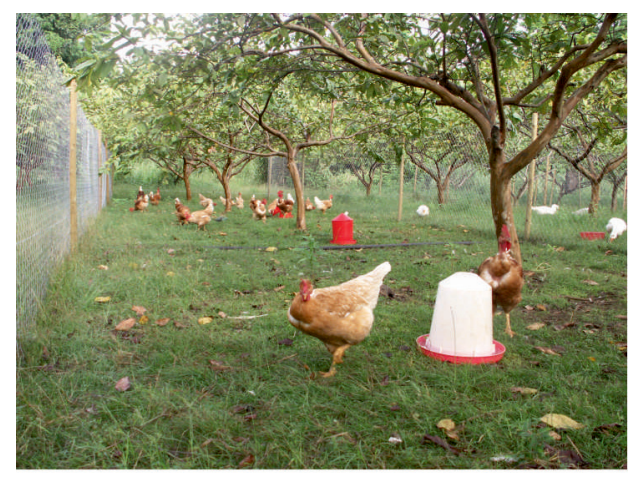

Figure 1.

Verger de goyaviers pâturé par des poulets (Gallus gallus) de la race Cou-nu rouge (Martinique).

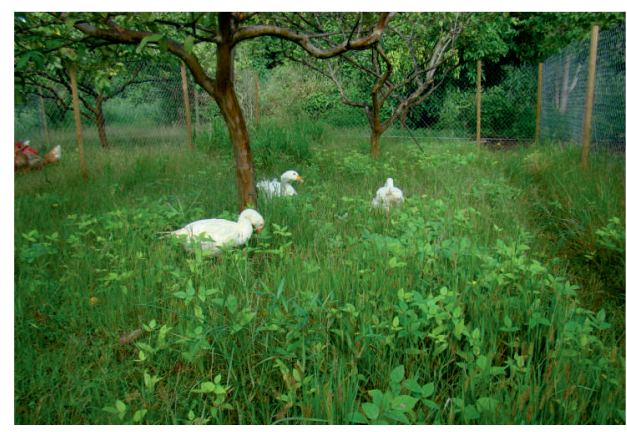

Figure 2.

Verger de goyaviers pâturé par des oies (Anser anser) de la race Blanche du Rhin (Martinique). et plantés à une densité de 400 plants par ha $(5 \mathrm{~m} \times 5 \mathrm{~m})$. Ce verger est naturellement enherbé et entretenu à la débroussailleuse.

Pour mener l'essai, nous avons subdivisé une parcelle de $700 \mathrm{~m}^{2}$ en sept placettes de $100 \mathrm{~m}^{2}$. Deux placettes ont constitué les placettes témoins; elles ont été fauchées régulièrement par l'agriculteur lorsque l'enherbement devenait gênant pour la réalisation des tâches courantes (la récolte notamment), soit deux fois au cours de notre essai. Sur les cinq autres placettes, nous avons fait pâturer vingt poulets ( $G a l$ lus gallus), de la race Cou-nu rouge (figure 1), et cinq oies (Anser anser), de la race Blanche du Rhin (figure 2), mâles et femelles de chacune des espèces. La race de poulets Cou-nu rouge a été choisie pour sa rusticité connue et appréciée localement ; la race d'oies Blanche du Rhin, pour ses qualités d'animal à viande et sa disponibilité chez les fournisseurs locaux. Les cinq placettes soumises à pâturage ont été suivies sur trois cycles de 5 semaines, pendant 15 semaines : chacune de ces placettes a été pâturée par les 5 oies pendant 1 semaine, puis par les 20 poulets la semaine suivante, 


\section{A. Lavigne et al.}

Tableau I.

Schéma de gestion d'un pâturage tournant par des oies et des poulets, en verger de goyaviers, sur sept placettes $\left(100 \mathrm{~m}^{2}\right)$ et pour un cycle de 5 semaines (Martinique).

\begin{tabular}{|c|c|c|c|c|c|c|}
\hline \multirow[t]{2}{*}{ Semaine } & \multicolumn{6}{|c|}{ Placette } \\
\hline & 1 & 2 & 3 & 4 & 5 & 6 \\
\hline 1 & Oies (5) & Sans pâturage & Sans pâturage & Sans pâturage & Poulets (20) & \multirow{5}{*}{$\begin{array}{l}\text { Placettes témoins: } \\
\text { Désherbage mécanique }\end{array}$} \\
\hline 2 & Poulets (20) & Oies (5) & Sans pâturage & Sans pâturage & Sans pâturage & \\
\hline 3 & Sans pâturage & Poulets (20) & Oies (5) & Sans pâturage & Sans pâturage & \\
\hline 4 & Sans pâturage & Sans pâturage & Poulets (20) & Oies (5) & Sans pâturage & \\
\hline 5 & Sans pâturage & Sans pâturage & Sans pâturage & Poulets (20) & Oies (5) & \\
\hline
\end{tabular}

puis laissées en repos les trois autres semaines (tableau I).

Les volailles étaient âgées de 15 semaines à leur entrée dans le dispositif. À cet âge, les poulets sont alors quasiment adultes alors que les oies ne le sont pas. L'entrée en ponte a lieu en effet à 17 semaines pour les poules et 36 semaines pour les oies, et l'âge d'abattage, qui varie selon les races et méthodes d'élevage, est au minimum de 12 semaines en agriculture biologique pour les poulets et de 20 semaines pour les oies.

Pour protéger les volailles des prédateurs (les chiens plus particulièrement), les cinq placettes pâturées ont été délimitées par un grillage de 1,50 $\mathrm{m}$ de hauteur; l'ensemble du dispositif a été protégé par une clôture électrifiée, branchée sur secteur, et les animaux ont été placés en poulailler chaque soir [10, 16], chaque espèce de volailles disposant d'un tel abri en bordure de parcelle.

Chaque animal, poulet ou oie, a reçu une ration réduite à $100 \mathrm{~g}$ par jour d'aliment complet du commerce, permettant de couvrir les besoins minimaux d'entretien, c'està-dire d'assurer les fonctions de base de l'organisme, mais insuffisante pour assurer leur satiété, cela pour inciter les animaux à pâturer.

\section{2. Échantillonnage}

La biomasse herbacée a été mesurée chaque semaine sur chacune des sept pla- cettes. À chaque date de mesure, quatre échantillons, soit quatre répétitions, ont été prélevés sur chaque placette, à l'aide d'un quadrat de $25 \mathrm{~cm}$ de côté. Pour chaque échantillon, cette biomasse a été séparée en trois catégories en fonction des familles végétales : poacées, cypéracées et dicotylédones.

Un inventaire floristique a été réalisé en début et en fin d'essai [19]. Une fois par mois, l'indice de recouvrement a été relevé pour chaque groupe botanique en utilisant une échelle de notation s'inspirant de la norme CEB [20].

\subsection{Analyse des résultats}

Une moyenne des mesures hebdomadaires de la biomasse herbacée des cinq placettes mises à pâturer par les volailles donne une information sur le taux de contrôle de l'enherbement de toute la parcelle au cours du temps.

Une moyenne des mesures hebdomadaires de chacune des placettes après le pâturage des oies, puis des poulets (cinq répétitions par rotation) indique l'effet des oies, et celui des poulets, sur la biomasse herbacée et la composition de l'enherbement.

L'effet du pâturage par les poulets et par les oies sur la biomasse herbacée a été testé par utilisation du test W de Wilcoxon apparié, à l'aide du logiciel R [21]. 


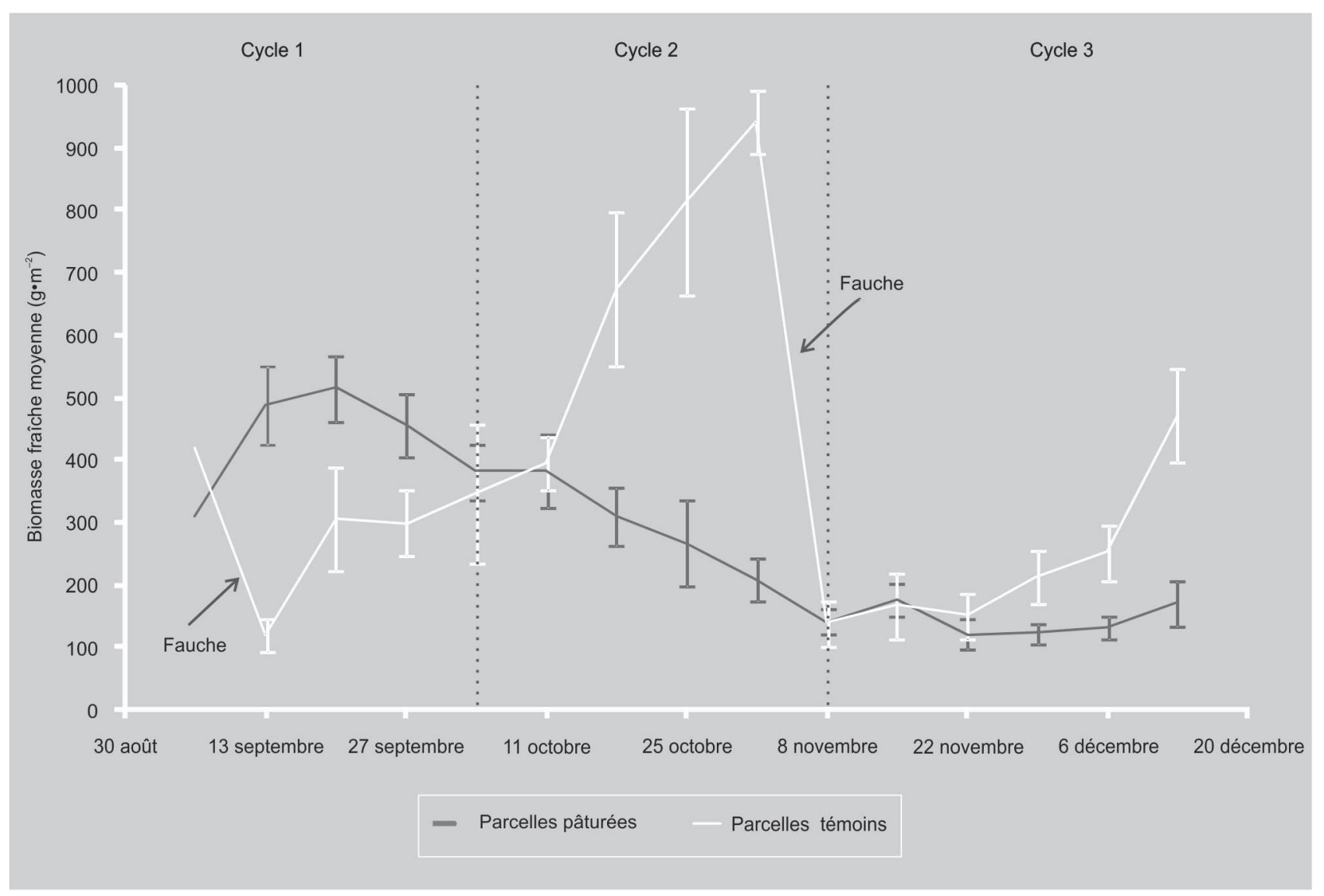

\section{Résultats}

\subsection{La biomasse de la strate herbacée diminue sur les placettes pâturées}

Sur les deux placettes témoins, l'enherbement a augmenté constamment après la fauche. La biomasse maximale atteinte est celle qui est supportable par l'agriculteur [(900 à 1000) $\left.\mathrm{g} \cdot \mathrm{m}^{-2}\right]$ : une fois cette valeur obtenue, la fauche devient nécessaire (figure 3). En revanche, l'enherbement dans les placettes pâturées n'a jamais atteint ce maximum. A contrario, la biomasse moyenne des cinq placettes pâturées a constamment diminué au cours de l'essai, jusqu'à atteindre un palier après le deuxième cycle (figure 3). Ce palier correspond aux valeurs de biomasses atteintes après la fauche sur les placettes témoins (13 septembre et 8 novembre 2010). La biomasse herbacée des placettes pâturées a donc peu augmenté pendant les trois semaines de repos (figure 4). La biomasse ne s'est pas rétablie entre deux cycles de pâturage.

Le pâturage par les volailles a diminué significativement la biomasse herbacée. Les oies ont un effet plus significatif que les poulets (test de Wilcoxon, $P<0,5$, tableau II). En comparant la biomasse moyenne des placettes pendant les trois cycles après pâturage par les oies, puis par les poulets, ou après un repos de 1,2 ou 3 semaines, on constate que les oies consomment la plus grande partie de la biomasse disponible, et que les poulets ont moins d'effet sur la biomasse totale (figure 4).

\subsection{Les volailles modifient la composition du pâturage}

L'inventaire floristique effectué en début d'essai, avant introduction des volailles, a

\section{Figure 3.}

Comparaison de l'évolution de la biomasse herbacée moyenne mesurée pendant quinze semaines sur cinq placettes soumises au pâturage de volailles (oies et poulets) et sur deux parcelles témoins non pâturées mais fauchées : les volailles diminuent la biomasse herbacée sur les placettes pâturées (les barres d'erreur représentent l'erreur standard). 


\section{A. Lavigne et al.}

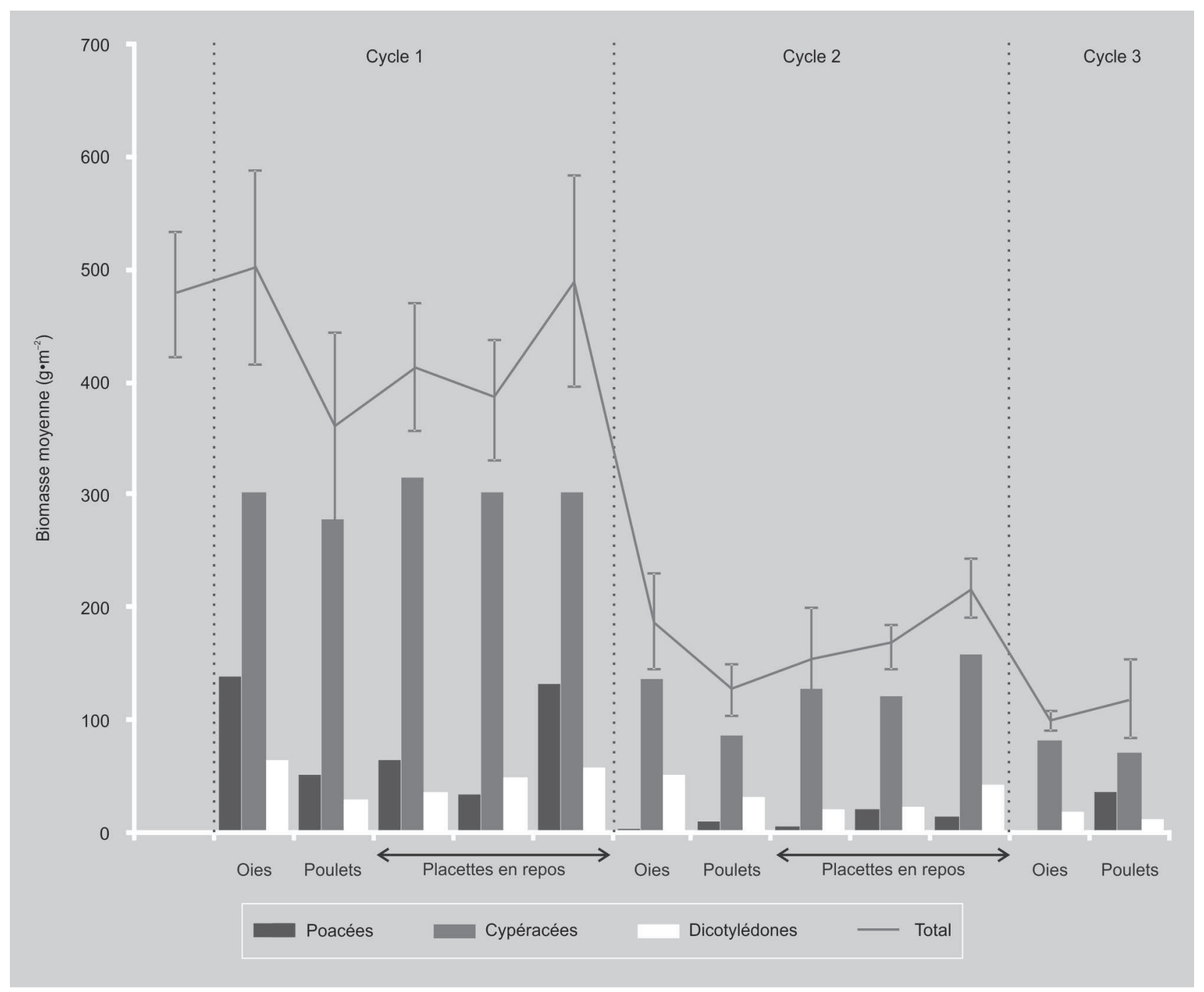

Figure 4.

Évolution de la biomasse moyenne obtenue (+ erreur standard) par trois groupes botaniques, sur cinq placettes expérimentales, après pâturage par des oies, des poulets, puis sans volaille, au cours de trois cycles de rotation : les oies consomment la plus grande partie de la biomasse disponible.
Tableau II.

Le pâturage par les volailles (oies et poulets) en verger de goyaviers martiniquais diminue la biomasse herbacée : résultats analysés à l'aide du test non paramétrique apparié de Wilcoxon.

\begin{tabular}{lccc} 
Volaille & $W$ & Valeur de $p$ & Significativité \\
\hline Poulets & 975.5 & 0.004598 & $p<0,05$ \\
Oies & 1215.5 & $8.327 e-07$ & $p<0,001$
\end{tabular}

permis d'inventorier 26 espèces végétales réparties en 11 familles botaniques. Les familles présentant les plus grandes richesses spécifiques étaient représentées

par les poacées $(12 / 267)$ et les cypéracées (3/26) constituées en grande partie d'espèces annuelles ou bisannuelles (tableau III). 
Tableau III.

Inventaire floristique et indices de recouvrement des espèces échantillonnées en verger de goyaviers martiniquais sur des placettes expérimentales pâturées par des volailles (oies, puis poulets). Mise en évidence de l'action sélective exercée par les volailles.

\begin{tabular}{|c|c|c|c|c|}
\hline Famille & Espèce & $\begin{array}{l}\text { Indice de recouvrement avant } \\
\text { introduction des volailles }\end{array}$ & $\begin{array}{l}\text { Indice de recouvrement en fin } \\
\text { d'expérimentation }\end{array}$ & $\begin{array}{l}\text { Évolution du } \\
\text { recouvrement }\end{array}$ \\
\hline Asteraceae & $\begin{array}{l}\text { Vernonia cinerea } \\
\text { Mikania micrantha }\end{array}$ & $<15 \%$ & $<15 \%$ & Stable \\
\hline Araceae & Caladium sp. & $<15 \%$ & Espèce non présente & Diminution \\
\hline Capparaceae & Cleome ruditosperma & $<15 \%$ & $<15 \%$ & Stable \\
\hline Cucurbitaceae & Momordica charantia & $<15 \%$ & Espèce non présente & Diminution \\
\hline Cyperaceae & $\begin{array}{l}\text { Cyperus sphacelatus } \\
\text { Kyllinga sp. } \\
\text { Fimbristylis sp. }\end{array}$ & $50 \%$ & $70 \%<$ recouvrement $<85 \%$ & Forte augmentation \\
\hline Euphorbiaceae & $\begin{array}{l}\text { Phyllanthus urinaria } \\
\text { Chamaesyce hirta }\end{array}$ & $<15 \%$ & $<15 \%$ & Stable \\
\hline Melastomataceae & Clidemia hirta & $<15 \%$ & $<15 \%$ & Stable \\
\hline Onagraceae & Ludwigia sp. & $<15 \%$ & $<15 \%$ & Stable \\
\hline Poaceae & $\begin{array}{l}\text { Setaria geniculata } \\
\text { Echinochloa colona } \\
\text { Digitaria violescens } \\
\text { Digitaria radicosa } \\
\text { Digitaria ciliaris } \\
\text { Eleusine indica } \\
\text { Cynodon dactylon } \\
\text { Sporobolus sp. } \\
\text { Chloris radiata } \\
\text { Axonopus compressus } \\
\text { Paspalum paniculatum } \\
\text { Leptochloa filiformis }\end{array}$ & $15 \%<$ recouvrement < $30 \%$ & Espèces non présentes & Forte diminution \\
\hline Rubiaceae & Hedyotis corymbosa & $<15 \%$ & Espèce non présente & Diminution \\
\hline Scrophulariaceae & Lindernia rotundifolia & $15 \%<$ recouvrement $<30 \%$ & $15 \%<$ recouvrement $<30 \%$ & Stable \\
\hline
\end{tabular}

Le pâturage a induit une modification progressive du couvert conduisant à la disparition de certaines espèces (poacées et quelques dicotylédones) et à une homogénéisation de la flore par l'augmentation du recouvrement des cypéracées. Toutefois certaines espèces se sont maintenues avec des indices de recouvrement faibles (Vernonia cinerea, Phyllanthus urinaria, etc.). En fin d'essai, seules onze espèces appartenant à sept familles botaniques ont été recensées (tableau III).

Sur les deux placettes témoins, une augmentation constante de toutes les espèces a été constatée après chaque opération de fauche, avec des valeurs de biomasse herbacée plus élevées pour les cypéracées (figure 5). Sur les placettes pâturées, au 


\section{A. Lavigne et al.}

Figure 5.

Évolution de la biomasse des poacées, cypéracées et dicotylédones sur des placettes expérimentales soumises à pâturage par des volailles (contrôle de l'enherbement) ou non pâturées (parcelles témoins, désherbage par fauchage).

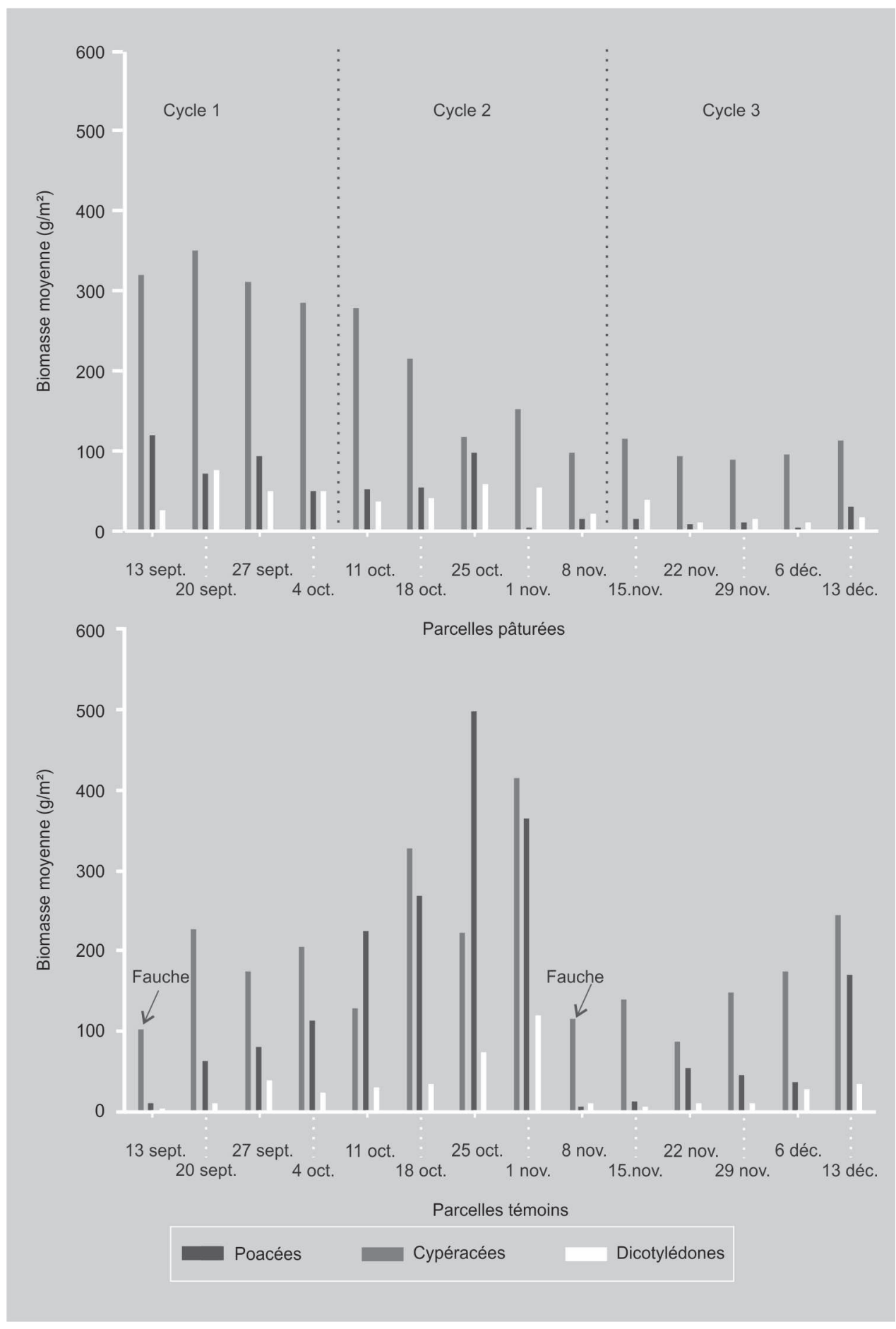

cours du deuxième cycle de pâturage, la biomasse des poacées a fortement diminué ; elle est restée très faible au cours du troisième cycle de pâturage. La biomasse des cypéracées a diminué également au cours des premier et deuxième cycles et est restée constante au cours du troisième cycle, quoique toujours supérieure à celle 
des poacées (figure 5). Les biomasses spécifiques obtenues sur les placettes pâturées en fin d'essai sont similaires aux biomasses obtenues après la fauche (figure 5).

Oies et poulets n'ont pas eu le même effet sur la composition du couvert (figure 4). La biomasse des dicotylédones après pâturage ou après repos des placettes a peu évolué. Après pâturage par les oies, les biomasses des cypéracées et des poacées ont fortement diminué. Les poacées ont complètement disparu lors du deuxième cycle de pâturage après passage des oies. En revanche, le pâturage par les poulets a peu modifié la composition spécifique du couvert, mais il a permis une légère repousse des poacées lors des deuxième et troisième cycles (figure 4).

\section{Discussion}

Le pâturage par les volailles, oies et poulets dans notre essai, a entrainé une diminution significative de la biomasse herbacée présente sur le verger de goyaviers étudié. Dès le deuxième passage des oies, cette biomasse est devenue équivalente à celle obtenue après une fauche à la débroussailleuse. Cette diminution de la biomasse s'est révélée davantage imputable au pâturage par les oies qu'à celui par les poulets. Une semblable efficacité des oies avait déjà été observée en verger de pommiers en Amérique du Nord [13]. La moindre diminution de biomasse constatée en verger de goyaviers après pâturage par les poulets pourrait être due à une charge trop faible en animaux : les poulets ne seraient réellement efficaces qu'à de fortes densités [10], et sur des surfaces plus restreintes aptes à les maintenir à proximité du poulailler [11, 22]

La biomasse herbacée des placettes a peu augmenté pendant les trois semaines de repos (sans pâturage). Cette période de trois semaines sans présence d'animaux sur la placette n'a pas permis de compenser la biomasse consommée par les volailles qui, à leur retour sur la placette, ne retrouvent pas un niveau satisfaisant d'herbes appétibles.

L'agriculteur a estimé devoir faucher les placettes témoins après huit semaines, alors que les oies et les poulets contrôlaient l'enherbement des placettes données en pâturage pendant cette période. L'objectif recherché qui était de réduire au moins de moitié le nombre de fauches a donc été atteint, en incluant la fauche en fin d'expérimentation des espèces refusées par les volailles.

À l'inverse d'une fauche mécanique qui réduit de manière similaire la biomasse de toutes les espèces présentes, le pâturage par les volailles a induit une modification de la composition spécifique du couvert. La richesse spécifique a diminué du fait de la disparition de certaines espèces (principalement les poacées mais aussi quelques dicotylédones) [23] et il y a eu une homogénéisation de la flore par augmentation du recouvrement par les cypéracées. Par conséquent, la pression exercée par la volaille sur l'enherbement se révèle beaucoup plus forte sur les poacées et les dicotylédones que sur les cypéracées. De précédents travaux avaient montré que les poacées étaient plus appétibles pour les oies que les autres plantes [13, 16] ; cela a conduit à une modification de la composition du couvert : dans les parcelles pâturées par les oies, le recouvrement par des espèces peu ou pas appétibles a augmenté rapidement dès les deux premières semaines de l'essai [16]. Une trop forte densité animale a pu amplifier le phénomène de sélection. En effet, le surpâturage, comme le pâturage sélectif, peut induire une modification de l'enherbement [24]. Cela signifierait que la charge animale par unité de surface pourrait être trop importante ou que la gestion du pâturage tournant (temps de pâturage et temps de repos) est mal adaptée à la repousse des poacées. Enfin, d'après Chicouène, un pâturage continu favoriserait le développement de plantes naines et le piétinement sélectionnerait le maintien des espèces coriaces [25]. Ainsi s'expliquerait le développement des cypéracées, les espèces de cette famille présentes sur les placettes étant à la fois de petite taille et coriaces.

Le pâturage des oies a permis de diminuer significativement la biomasse herbacée des plantes appétibles. Si l'établissement d'un système durable est l'objectif visé et 
que la flore du verger est composée de beaucoup d'espèces non appétibles, le pâturage par les oies ne pourra constituer la seule méthode de désherbage [16] ; il devra être associé à d'autres techniques destinées à mâtriser les espèces non appétibles.

À la différence des oies, les poulets ont consommé les fruits tombés sur le sol. Ils ont ainsi participé au maintien du bon état sanitaire du verger de goyaviers en réduisant les populations de ravageurs tels les mouches des fruits [10, 12, 22].

Le système utilisé dans notre étude a donc bien permis de limiter la biomasse herbacée à un volume suffisamment faible pour permettre la réalisation des pratiques culturales par l'agriculteur. Le système tel qu'il a été présenté n'est cependant pas pérenne : sans autres interventions, les espèces moins consommées par les volailles devraient continuer à prendre de l'importance. Pour être efficace à long terme, le système devra être modifié. D'après Clark, l'efficacité du pâturage par les volailles dépendrait de différents facteurs qui sont, entre autres, la densité animale, le temps de pâturage et la fréquence de rotation, ainsi que la composition de la flore herbacée présente [10, 13]. Plusieurs solutions pourraient donc être envisageables pour pérenniser le système : diminution de la charge animale par unité de surface, modification des modalités de rotation ou encore combinaison du pâturage avec une autre méthode de désherbage tel que le désherbage mécanique [7, 16]. On pourrait également envisager de ne pas placer les volailles sur un couvert spontané mais sur un mélange de plantes de couverture appétibles, installées préalablement. Ce couvert pourrait alors se maintenir grâce à une charge animale adéquate et une gestion correcte du temps de pâturage et de la fréquence de rotation des volailles.

L'utilisation des volailles, des oies en particulier, se révèle être une méthode efficace pour la maîtrise de l'enherbement, utilisable aussi bien en agriculture conventionnelle qu'en production biologique ; elle requiert cependant une adaptation technique en fonction de la flore du verger et un accompagnement des agriculteurs.
L'utilisation en vergers d'autres espèces de volailles, comme les canards de Barbarie et les pintades, moins sélectifs sur leur régime alimentaire, est en cours d'expérimentation chez d'autres producteurs.

\section{References}

[1] Ollagnier S., Vittecoq B., Suivi de la qualité des eaux souterraines de Martinique, campagne de saison des pluies 2006 : résultats et interprétation, BRGM/RP-55499-FR, BGRM, Martinique, France, 2007.

[2] Hipps N.A., Samuelson T.J., Effects of longterm herbicide use, irrigation and nitrogen fertiliser on soil fertility in an apple orchard, J. Sci. Food Agric. 55 (1991) 377-387.

[3] Duran Zuazo V.H., Rodriguez Pleguezuelo C.R., Soil-erosion and runoff prevention by plant covers. A review, Agron. Sustain. Dev. 28 (2008) 65-86.

[4] Brownlow M.J.C, Dorward P.T., Carruthers S.P., Integrating natural woodland with pig production in the United Kingdom: an investigation of potential performance and interactions, Agrofor. Syst. 64 (2005) 251-263.

[5] Gordon A.M., Newman S., Temperate agroforestry systems, CAB Int., Wallingford, U.K., 1997.

[6] Sharrow S.H., Silvopastoralism: competition and facilitation between trees, livestock, and improved grass-clover pastures on temperate rainfed lands, in: Buck L.E., Lassoie J.P., Fernandes E.C.M. (Eds.), Agroforestry in sustainable agricultural systems, Lewis Publ., N. Y., U.S.A., 1999.

[7] Kathiresan R.M, Integration of elements of a farming system for a sustainable weed and pest management in the tropics, Crop Prot. 26 (2007) 424-429.

[8] Gliessman S.R., Agroecology: the ecology of sustainable food systems, CRC Press, Boca Raton, U.S.A., 2006.

[9] Yates C., Dorward P., Hemery G., Cook P., The economic viability and potential of a novel poultry agroforestry system, Agrofor. Syst. 69 (2007) 13-28.

[10] Clark M.S., Gage S.H, Effects of free-range chickens and geese on insect pests and weeds in an agroecosystem, Am. J. Altern. Agric. 11 (1996) 39-47.

[11] Mayton E.L., Smith E.V., King D., Nutgrass eradication studies: IV. Use of chicken and 
geese in the control of nutgrass (Cyperus rotundus L.), J. Am. Soc. Agron. 47 (1945) 785-791.

[12] Hermansen J.E., Strudsholm K., Horsted K., Integration of organic animal production into land use with special reference to swine and poultry, Livestock Prod. Sci. 90 (2004) 11-26.

[13] Clark M.S., Gage S.H, DeLind L., Lennington M., The compatibility of domestic birds with a nonchemical agroecosystem, Am. J. Altern. Agric. 10 (1995) 114-121.

[14] Kasasian L., Weed control in cotton, Cotton Grow. Rev. 46 (1969) 165-173.

[15] Buckland R., Guy G., Production des oies. Production et santé animales, Étude FAO, no. 154, FAO, Rome, Italie, 2002.

[16] Wurtz T.L., Domestic geese: biological weed control in an agricultural setting, Ecol. Appl. 5 (1995) 570-578.

[17] Popay I., Field R., Grazing animals as weed control agents, Weed Technol. 10 (1996) 217-231.

[18] Colmet-Daage F., Carte des sols des Antilles au 1/20000, ORSTOM-Martinique, Antilles, Paris, France, 1969.

[19] Fournet J., Flore illustrée des phanérogames de Guadeloupe et de Martinique, CIRAD, Montpellier, France, 2002.
[20] Marnotte P., Influence des facteurs agroécologiques sur le développement des mauvaises herbes en climat tropical humide, in: Ile Colloq. Int. L'écologie, la biologie et la systématique des mauvaises herbes, COLUMAEWRS, Paris, France, 1984, pp. 183-189.

[21] Anon., R: A language and environment for statistical computing, R Dev. Core Team, R Found. Stat. Comput., Vienna, Austria, 2004.

[22] Pedersen L.H., Olsen A., Pedersen B., Korsgaard M., Horsted K., Combined production of broilers and fruits, in: Boos $\mathrm{M}$. (Ed.), Proc. Xlth Ecofruit, Int. Conf. Cultivation technique and phytopathological problems in organic fruit-growing, Weinsberg, Ger., 2004, pp. 131-136.

[23] Kruess A., Tscharntke T., Contrasting responses of plant and insect diversity to variation in grazing intensity, Biol. Conserv. 106 (2001) 293-302.

[24] Walker B.H., Stability in rangelands: ecology and economics, in: Baker M.J., Crush J.R., Humphrey L.R (Eds.), Proc. XVII7th Int. Grassl. Congr., Palmerst. N., N.Z., 1993, pp. 1885-1890.

[25] Chicouène D., La gestion par la fauche et le pâturage des milieux herbacés tempérés. Comment raisonner la description biologique de la flore ? Ing. 46 (2006) 51-66.

\section{Las aves de corral para un control biológico de los arvenses en huertas.}

Resumen - Introducción. Dadas las condiciones tropicales de humedad, el control de los arvenses es un tema importante y permanente en los gastos de un productor de frutas en las Antillas. La práctica del deshierbe químico es, a día de hoy, la más económica, pero el uso recurrente de herbicidas en los huertas de Martinica conduce a una erosión de los suelos y a una contaminación de las aguas. Para paliar el uso de herbicidas, hemos testado la asociación de aves de corral en un huerta productivo, con el fin de controlar el enyerbamiento. Material y métodos. Se instaló el dispositivo en una parcela de $700 \mathrm{~m}^{2}$, subdividida en siete parcelas de ensayo de $100 \mathrm{~m}^{2}$ cada una. Dos parcelas de ensayo constituyeron los tratamientos testigo: fueron segadas por medio de la desbrozadora. Cada una de las otras cinco parcelas de ensayo fue sometida al pasto de 5 ocas durante una semana, luego al pasto de pollos durante la semana posterior, luego se dejaron sin aves de corral durante otras tres semanas. Se observaron tres ciclos de tales rotaciones. Se midió la producción de biomasa por grupo botánico (poáceas, ciperáceas y otros) en las parcelas experimentales, cada semana, durante los tres ciclos ( 4 meses). Una vez al mes, se anotó el índice de recubrimiento por grupo botánico. Resultados. La biomasa total del estrato herbáceo disminuyó en las parcelas de ensayo pastadas, lo que permitió el control biológico de los arvenses durante cuatro meses. La diversidad florística disminuyó, a causa de la selección practicada por las aves de corral para su alimentación. Asimismo, la cubierta se homogeneizó, por el aumento del porcentaje de biomasa de las ciperáceas en la biomasa total. De ahí que esta familia, poco apetecible para las aves de corral, se extendiese por todas las parcelas de ensayo pastadas. Discusión. El pasto de las aves de corral, de las ocas en particular, ha resultado ser un método eficaz para gestionar el enyerbamiento en huerta. Sin embargo, en el caso de una fuerte proporción de especies no apetecibles en el estrato herbáceo, deberán combinarse otros métodos de deshierbe. Para optimizar esta técnica, deberá plantearse la instalación de una capa de hierba compuesta de una mezcla de vegetación de cubierta adaptada a la asociación huerta - aves de corral, así como una mejor gestión de la rotación de los animales.

Martinica / Psidium guajava / huerta frutal / plantas perennes / cultivo / lucha integrada / escarda / ganso / pollo 